\title{
Functional Limit Theorem for Products of Sums of Independent and Nonidentically Distributed Random Variables
}

\author{
Przemysław Matuła and Iwona Stępień \\ Institute of Mathematics, Maria Curie-Skłodowska University, Plac M.C.-Skłodowskiej 1, 20-031 Lublin, Poland \\ Correspondence should be addressed to Przemysław Matuła; matula@hektor.umcs.lublin.pl
}

Received 27 August 2012; Accepted 11 November 2012

Academic Editor: Inés Couso

Copyright (c) 2013 P. Matuła and I. Stępień. This is an open access article distributed under the Creative Commons Attribution License, which permits unrestricted use, distribution, and reproduction in any medium, provided the original work is properly cited.

We study the weak convergence in the space $D[0,1]$ of processes constructed from products of sums of independent but not necessarily identically distributed random variables. The presented results extend and generalize limit theorems known so far for i.i.d. sequences.

\section{Introduction}

Let $\left(X_{n}\right)_{n \in \mathbb{N}}$ be a sequence of independent, positive, and square-integrable random variables (r.v.s) defined on some probability space $(\Omega, \mathfrak{F}, P)$. For every $n \in \mathbb{N}$ let us put

$$
\begin{array}{ll}
\mu_{n}=E X_{n}, & \tau_{n}^{2}=\operatorname{Var}\left(X_{n}\right), \\
S_{n}=\sum_{k=1}^{n} X_{k}, & \sigma_{n}^{2}=\operatorname{Var}\left(S_{n}\right)=\sum_{k=1}^{n} \tau_{k}^{2} .
\end{array}
$$

Arnold and Villaseñor [1] obtained the central limit theorem for sums of records. Rempała and Wesołowski [2] observed that this result was of general nature and proved that for a sequence of i.i.d. positive and square-integrable r.v's one has

$$
\left(\frac{\prod_{i=1}^{n} S_{i}}{n ! \mu^{n}}\right)^{1 / \gamma \sqrt{n}} \stackrel{d}{\longrightarrow} e^{\sqrt{2} \cdot \mathcal{N}}, \quad \text { as } n \longrightarrow \infty
$$

where $\mathcal{N}$ is a standard normal variable and $\mu=\mu_{n}$ and $\gamma=\tau_{n} / \mu_{n}$ are the common mean and the coefficient of variation of the r.v.s. The convergence in (2) was generalized and extended by many authors in different ways. For example, Zhang and Huang [3] studied the functional version of this result. They proved under general conditions, not involving the dependence structure of the sequence, that

$$
\begin{aligned}
& \left(\frac{\prod_{i=1}^{[n t]} S_{i}}{n ! \mu^{n}}\right)^{1 / \gamma \sqrt{n}} \\
& \stackrel{d}{\longrightarrow} \exp \left(\int_{0}^{t} \frac{W(x)}{x} d x\right) \text { in } D[0,1] \text {, as } n \longrightarrow \infty,
\end{aligned}
$$

here and in the sequel $W(x)$ denotes the Wiener process. The functional version of the convergence of products of sums was also studied by Kosiński [4], for i.i.d. sequences belonging to the domain of attraction of the $\alpha$-stable law with $\alpha \in(1,2]$. The first result for sequences of nonidentically distributed r.v's was obtained by Matuła and Stępień [5]. Similarly as in the invariance principle, the study of the non-i.i.d. sequences requires defining the processes in a different way than in (3). For $t \in[0,1]$ and each $n \in \mathbb{N}$ let us define the function $m_{n}(t)=\max \left\{i \in \mathbb{N} \cup\{0\}: \sigma_{i}^{2} \leq t \sigma_{n}^{2}\right\}$, where $\sigma_{0}^{2}=0$. Let us introduce the process

$$
V_{n}(t):=\left(\prod_{i=1}^{m_{n}(t)}\left(\frac{S_{i}}{E S_{i}}\right)^{a_{i, n}}\right)^{1 / \sigma_{n}}
$$

and $V_{n}(t)=1$ for $t$ such that $m_{n}(t)=0$, where $a_{i, n}=$ $f\left(\sigma_{i}^{2} / \sigma_{n}^{2}\right)\left(\tau_{i+1}^{2} / \sigma_{n}^{2}\right) E S_{i}$. In [5] it was proved that for a sequence of independent, positive, and square-integrable random 
variables satisfying the Lindeberg condition and such that $\sum_{i=1}^{\infty} \tau_{i+1}^{2} /\left(E S_{i}\right)^{2}<\infty$, the process $V_{n}(t)$ converges weakly in the space $D[0,1]$ to the process $\exp \left(\int_{0}^{t} f(x) W(x) d x\right)$. This result was proved in one theorem for the function $f(x)=1 / x$ and in the second for functions $f \in C^{1}[0,1]$.

Our goal is to prove the aforementioned result for a large class of continuous functions on $(0,1]$, which may be unbounded. Furthermore, we shall slightly change the process $V_{n}(t)$ in order to avoid its artificial definition in the case $m_{n}(t)=0$ and replace the strange shifted index in $\tau_{i+1}^{2}$. In this setting, the imposed conditions and obtained results will be much more natural.

\section{Main Results}

Let $\mathscr{H}$ be the family of functions $f:[0,1] \rightarrow \mathbb{R}$ which are continuously differentiable on $(0,1]$, that is, $f \in \mathscr{C}^{1}(0,1]$, furthermore, such that

$$
\begin{aligned}
& \int_{0}^{1}|f(x)| \sqrt{x \log \left(\frac{1}{x}\right)} d x<\infty, \\
& f(x) x^{3 / 2} \longrightarrow 0, \quad \text { as } x \longrightarrow 0^{+} .
\end{aligned}
$$

Let us observe that from (5) it follows that $f(x) \sqrt{x}$ is integrable, if this function is also monotonic then, in consequence, (6) is satisfied. It is obvious that $\mathscr{C}^{1}[0,1] \subset \mathscr{H}$. The family $\mathscr{H}$ also contains functions of the form $f(x)=x^{-p}$ with $p \in(0,3 / 2)$ and $f(x)=1 /\left(x^{3 / 2} \log ^{r}(1 / x \wedge\right.$ e $\left.)\right)$ with $r>3 / 2$ and the value at 0 is arbitrary.

For any $n \in \mathbb{N}$ let us define the process

$$
T_{n}(t):=\left(\prod_{i=1}^{m_{n}(t)+1}\left(\frac{S_{i}}{E S_{i}}\right)^{\alpha_{i, n}}\right)^{1 / \sigma_{n}},
$$

where $\alpha_{i, n}=f\left(\sigma_{i}^{2} / \sigma_{n}^{2}\right)\left(\tau_{i}^{2} / \sigma_{n}^{2}\right) E S_{i}$ and we also take $m_{n}(1)=$ $n-1$. In this way we obtain a sequence of processes in $D[0,1]$ such that

$$
\begin{gathered}
T_{n}(t)=\left(\prod_{i=1}^{k}\left(\frac{S_{i}}{E S_{i}}\right)^{\alpha_{i, n}}\right)^{1 / \sigma_{n}}, \\
\text { for } t \in\left\langle\frac{\sigma_{k-1}^{2}}{\sigma_{n}^{2}}, \frac{\sigma_{k}^{2}}{\sigma_{n}^{2}}\right), \quad k=1,2, \ldots, n-1, \\
T_{n}(t)=\left(\prod_{i=1}^{n}\left(\frac{S_{i}}{E S_{i}}\right)^{\alpha_{i, n}}\right)^{1 / \sigma_{n}}, \\
\text { for } t \in\left\langle\frac{\sigma_{n-1}^{2}}{\sigma_{n}^{2}}, 1\right\rangle,
\end{gathered}
$$

recall that we have defined $\sigma_{0}^{2}=0$. Under our assumptions the trajectories of the process $T_{n}(t)$ are almost surely positive functions.

Our main result concerning weak convergence of $T_{n}(t)$ in the space $D[0,1]$ is as follows.
Theorem 1. Let $\left(X_{n}\right)_{n \in \mathbb{N}}$ be a sequence of independent, positive, and square-integrable r.v.s satisfying the Lindeberg condition:

$$
\lim _{n \rightarrow \infty} \frac{1}{\sigma_{n}^{2}} \sum_{i=1}^{n} E\left(\left(X_{i}-E X_{i}\right)^{2} \mathbf{I}\left[\left|X_{i}-E X_{i}\right| \geq \varepsilon \sigma_{n}\right]\right)=0,
$$

$$
\forall \varepsilon>0,
$$

and such that

$$
E S_{n} \longrightarrow \infty, \quad \text { as } n \longrightarrow \infty, \sum_{i=1}^{\infty} \frac{\tau_{i}^{2}}{\left(E S_{i}\right)^{2}}<\infty .
$$

Then for any $f \in \mathscr{H}$

$$
\begin{array}{r}
T_{n}(t) \stackrel{d}{\longrightarrow} \exp \left(\int_{0}^{t} f(x) W(x) d x\right) \quad \text { in } D[0,1], \\
\text { as } n \longrightarrow \infty .
\end{array}
$$

In order to illustrate our result let us present two examples.

Example 2. Let $\left(\xi_{k}\right)_{k \in \mathbb{N}}$ be a sequence of independent r.v's with Poisson distribution $\operatorname{Po}\left(\lambda_{k}\right)$ with parameter $\lambda_{k}=k$. Let us take $X_{k}=\xi_{k}+1$, then $X_{k} \geq 1$ almost surely, $E X_{k}=k+1$ and $\tau_{k}^{2}=\operatorname{Var}\left(X_{k}\right)=k$. Moreover, $E S_{n}=2+\cdots+(n+1)=n(n+$ 3) $/ 2$ and $\sigma_{n}^{2}=\operatorname{Var} S_{n}=1+\cdots+n=n(n+1) / 2$. Let us observe that $E\left|X_{k}-E X_{k}\right|^{3}=E\left|\xi_{k}-E \xi_{k}\right|^{3}$ but $\xi_{k}$ may be considered as a sum of i.i.d. (Poisson) r.v.s. Thus, from the well-known moment bounds, there exists a constant $C>0$ not depending on $k$ such that $E\left|\xi_{k}-E \xi_{k}\right|^{3} \leq C \cdot k^{3 / 2}$, for every $k \in \mathbb{N}$. Therefore, the Lyapunov condition may be easily verified. The condition (10) is also satisfied. We apply our Theorem 1 with $f(x)=1 / x$, then $\alpha_{i, n}=\left(\tau_{i}^{2} / \sigma_{i}^{2}\right) E S_{i}=i(i+3) /(i+1)$ and we have the following weak convergence in the space $D[0,1]$, as $n \rightarrow \infty$

$$
\begin{gathered}
\left(\prod_{i=1}^{m_{n}(t)+1}\left(\frac{2 S_{i}}{i(i+3)}\right)^{i(i+3) /(i+1)}\right)^{1 / \sqrt{n(n+1) / 2}} \\
\stackrel{d}{\longrightarrow} \exp \left(\int_{0}^{t} \frac{W(x)}{x} d x\right) .
\end{gathered}
$$

It may be easily checked (see page 42 in [6] for details) that the integral $\int_{0}^{1} x^{p} W(x) d x$ has Gaussian distribution $\mathscr{N}\left(0, \sigma^{2}\right)$ with

$$
\sigma^{2}=\iint_{0}^{1} \min (x, y) x^{p} y^{p} d x d y=\frac{2}{(p+2)(2 p+3)},
$$

provided $p>-3 / 2$. Therefore, we have the following example.

Example 3. Let $\left(X_{k}\right)_{k \in \mathbb{N}}$ be a sequence of i.i.d. r.v.s with the standard exponential distribution. Then $E X_{k}=\operatorname{Var}\left(X_{k}\right)=1$ and $\sigma_{n}^{2}=n$. We apply Theorem 1 with $t=1$ and the function 
$f(x)=x^{p}$, where $p>-3 / 2$. In this case $\alpha_{i, n}=(i / n)^{p+1}$ and we get

$$
\begin{array}{r}
\left(\prod_{i=1}^{n}\left(\frac{S_{i}}{i}\right)^{i^{p+1}}\right)^{1 / n^{(p+3 / 2)}} \stackrel{d}{\longrightarrow} \exp \left(\sqrt{\frac{2}{(p+2)(2 p+3)}} \mathcal{N}\right), \\
\text { as } n \longrightarrow \infty .
\end{array}
$$

\section{Proofs and Auxiliary Results}

In the proof of our main result, we shall use the following lemma.

Lemma 4. Let $d_{D[0,1]}$ be the metric making the space $D[0,1]$ to be a complete and separable metric space (Polish space) and let $d_{U}(x(t), y(t))=\sup _{0 \leq t \leq 1}|x(t)-y(t)|$ be the uniform metric. Furthermore, denote by $d_{L P D[0,1]}$ the Lévy-Prohorov metric in the space of probability measures on $D[0,1]$ and by $d_{K F D[0,1]}$ the Ky-Fan metric. Let $X, Y$, and $X_{n}$ be random elements with values in $D[0,1]$, then

(a) if $E d_{U}(X, Y) \leq a$, then $d_{K F D[0,1]}(X, Y) \leq \sqrt{a}$,

(b) $d_{L P D[0,1]}(X, Y) \leq d_{K F D[0,1]}(X, Y)$,

(c) if $d_{U}\left(X_{n}, X\right) \rightarrow 0$ almost surely, then $d_{K F D[0,1]}$ $\left(X_{n}, X\right) \rightarrow 0$.

Proof. The proof is based on the inequality $d_{D[0,1]} \leq d_{U}$, which may be found on page 150 in [7]. The inequality (b) is proved, for example, in Theorem 11.3.5 on page 397 in [8].

Proof of Theorem 1. In the first step we shall prove that

$$
\log T_{n}(t)=\frac{1}{\sigma_{n}} \sum_{i=1}^{m_{n}(t)+1} f\left(\frac{\sigma_{i}^{2}}{\sigma_{n}^{2}}\right) \frac{\tau_{i}^{2}}{\sigma_{n}^{2}}\left(S_{i}-E S_{i}\right)+A_{n}(t),
$$

where $A_{n} \stackrel{P}{\rightarrow} 0$ in $D[0,1]$, as $n \rightarrow \infty$. We shall apply the expansion of the logarithm $\log (1+x)=x+R(x)$, for $|x| \leq$ $1 / 2$, where $|R(x)| \leq 2 x^{2}$. Let us put $C_{i}=\left(S_{i}-E S_{i}\right) / E S_{i}$, then we easily get

$$
\begin{aligned}
\log \left(\frac{S_{i}}{E S_{i}}\right)= & \log \left(C_{i}+1\right) \\
= & C_{i}+R\left(C_{i}\right) \mathbf{I}\left[\left|C_{i}\right| \leq \frac{1}{2}\right]+\left[\log \left(1+C_{i}\right)-C_{i}\right] \\
& \times \mathbf{I}\left[\left|C_{i}\right|>\frac{1}{2}\right]
\end{aligned}
$$

$$
\begin{aligned}
\log T_{n}(t)= & \frac{1}{\sigma_{n}} \sum_{i=1}^{m_{n}(t)+1} f\left(\frac{\sigma_{i}^{2}}{\sigma_{n}^{2}}\right) \frac{\tau_{i}^{2} E S_{i}}{\sigma_{n}^{2}} \log \left(\frac{S_{i}}{E S_{i}}\right) \\
= & \frac{1}{\sigma_{n}} \sum_{i=1}^{m_{n}(t)+1} f\left(\frac{\sigma_{i}^{2}}{\sigma_{n}^{2}}\right) \frac{\tau_{i}^{2} E S_{i}}{\sigma_{n}^{2}} C_{i} \\
& +\frac{1}{\sigma_{n}} \sum_{i=1}^{m_{n}(t)+1} f\left(\frac{\sigma_{i}^{2}}{\sigma_{n}^{2}}\right) \frac{\tau_{i}^{2} E S_{i}}{\sigma_{n}^{2}} R\left(C_{i}\right) \mathbf{I}\left[\left|C_{i}\right| \leq \frac{1}{2}\right] \\
& +\frac{1}{\sigma_{n}} \sum_{i=1}^{m_{n}(t)+1} f\left(\frac{\sigma_{i}^{2}}{\sigma_{n}^{2}}\right) \frac{\tau_{i}^{2} E S_{i}}{\sigma_{n}^{2}}\left[\log \left(1+C_{i}\right)-C_{i}\right] \\
& \times \mathbf{I}\left[\left|C_{i}\right|>\frac{1}{2}\right] \\
= & \frac{1}{\sigma_{n}} \sum_{i=1}^{m_{n}(t)+1} f\left(\frac{\sigma_{i}^{2}}{\sigma_{n}^{2}}\right) \frac{\tau_{i}^{2}}{\sigma_{n}^{2}}\left(S_{i}-E S_{i}\right)+A_{n}^{\prime}(t) \\
& +A_{n}^{\prime \prime}(t), \text { say. }
\end{aligned}
$$

Let us observe that

$$
\begin{aligned}
& E \max _{0 \leq t \leq 1}\left|A_{n}^{\prime}(t)\right| \\
& \quad \leq E \frac{1}{\sigma_{n}} \sum_{i=1}^{n}\left|f\left(\frac{\sigma_{i}^{2}}{\sigma_{n}^{2}}\right)\right| \frac{\tau_{i}^{2} E S_{i}}{\sigma_{n}^{2}}\left|R\left(C_{i}\right)\right| \mathbf{I}\left[\left|C_{i}\right| \leq \frac{1}{2}\right] \\
& \quad \leq \frac{2}{\sigma_{n}} \sum_{i=1}^{n}\left|f\left(\frac{\sigma_{i}^{2}}{\sigma_{n}^{2}}\right)\right| \frac{\tau_{i}^{2} E S_{i}}{\sigma_{n}^{2}} E\left(\frac{S_{i}-E S_{i}}{E S_{i}}\right)^{2} \\
& =2 \sum_{i=1}^{n}\left|f\left(\frac{\sigma_{i}^{2}}{\sigma_{n}^{2}}\right)\right| \frac{\sigma_{i}}{\sigma_{n}} \frac{\tau_{i}^{2}}{\sigma_{n}^{2}} \frac{\sigma_{i}}{E S_{i}} .
\end{aligned}
$$

From the assumption (10) and the Kronecker lemma it follows that $\left(1 /\left(E S_{n}\right)^{2}\right) \sum_{i=1}^{n} \tau_{i}^{2}=\sigma_{n}^{2} /\left(E S_{n}\right)^{2} \rightarrow 0$. It is easy to see that from the Lindeberg condition (9), the Feller condition

$$
\lim _{n \rightarrow \infty} \max _{1 \leq i \leq n} \frac{\tau_{i}^{2}}{\sigma_{n}^{2}}=0
$$

holds; therefore,

$$
\sum_{i=1}^{n}\left|f\left(\frac{\sigma_{i}^{2}}{\sigma_{n}^{2}}\right)\right| \frac{\sigma_{i}}{\sigma_{n}} \frac{\tau_{i}^{2}}{\sigma_{n}^{2}} \longrightarrow \int_{0}^{1}|f(x)| \sqrt{x} d x<\infty .
$$

Moreover, by our assumption (6) we get

$$
\left|f\left(\frac{\sigma_{i}^{2}}{\sigma_{n}^{2}}\right)\right| \frac{\sigma_{i}}{\sigma_{n}} \frac{\tau_{i}^{2}}{\sigma_{n}^{2}} \leq\left|f\left(\frac{\sigma_{i}^{2}}{\sigma_{n}^{2}}\right)\right|\left(\frac{\sigma_{i}}{\sigma_{n}}\right)^{3} \longrightarrow 0 .
$$

By the above remarks and Toeplitz theorem on the transformation of sequences into sequences (Problem 2.3.1 in [9]) we obtain

$$
\sum_{i=1}^{n}\left|f\left(\frac{\sigma_{i}^{2}}{\sigma_{n}^{2}}\right)\right| \frac{\sigma_{i}}{\sigma_{n}} \frac{\tau_{i}^{2}}{\sigma_{n}^{2}} \frac{\sigma_{i}}{E S_{i}} \longrightarrow 0
$$

Thus from (17) we have $A_{n}^{\prime} \stackrel{P}{\rightarrow} 0$ in $D[0,1]$, as $n \rightarrow \infty$. 
From (10) the strong law of large numbers holds, that is, $C_{n}=\left(S_{n}-E S_{n}\right) / E S_{n} \rightarrow 0$ almost surely. Thus, for almost all $\omega \in \Omega$ and sufficiently large $i \geq i_{0}(\omega)$ there holds $\mathbf{I}\left[\left|C_{i}(\omega)\right|>\right.$ $1 / 2]=0$. Since that

$$
\begin{aligned}
\max _{0 \leq t \leq 1} & \left|A_{n}^{\prime \prime}(t)\right| \\
\leq & \frac{1}{\sigma_{n}} \sum_{i=1}^{n}\left|f\left(\frac{\sigma_{i}^{2}}{\sigma_{n}^{2}}\right)\right| \frac{\tau_{i}^{2} E S_{i}}{\sigma_{i}^{2}}\left|\log \left(1+C_{i}\right)-C_{i}\right| \\
& \times \mathbf{I}\left[\left|C_{i}\right|>\frac{1}{2}\right] \longrightarrow 0, \text { a.s. }
\end{aligned}
$$

Consequently $A_{n}^{\prime \prime} \stackrel{P}{\rightarrow} 0$ in $D[0,1]$, and (15) is proved.

It remains to prove that

$$
\begin{gathered}
Y_{n}(t):=\frac{1}{\sigma_{n}} \sum_{i=1}^{m_{n}(t)+1} f\left(\frac{\sigma_{i}^{2}}{\sigma_{n}^{2}}\right) \frac{\tau_{i}^{2}}{\sigma_{n}^{2}}\left(S_{i}-E S_{i}\right) \\
\stackrel{d}{\longrightarrow} \int_{0}^{t} f(x) W(x) d x
\end{gathered}
$$

in $D[0,1]$, as $n \rightarrow \infty$.

Let $0<\varepsilon<1$ be given and let us define a process

$$
\begin{aligned}
& Y_{n, \varepsilon}(t) \\
& \quad= \begin{cases}0, & 0 \leq t<\frac{\sigma_{m_{n}(\varepsilon)}^{2}}{\sigma_{n}^{2}} \\
\frac{1}{\sigma_{n}} \sum_{i=m_{n}(\varepsilon)+1}^{m_{n}(t)+1} f\left(\frac{\sigma_{i}^{2}}{\sigma_{n}^{2}}\right) \frac{\tau_{i}^{2}}{\sigma_{n}^{2}}\left(S_{i}-E S_{i}\right), & \frac{\sigma_{m_{n}(\varepsilon)}^{2}}{\sigma_{n}^{2}} \leq t \leq 1 .\end{cases}
\end{aligned}
$$

Let us observe that

$$
\begin{aligned}
& E \max _{0 \leq t \leq 1}\left|Y_{n}(t)-Y_{n, \varepsilon}(t)\right| \\
& \leq E \frac{1}{\sigma_{n}} \sum_{i=1}^{m_{n}(\varepsilon)}\left|f\left(\frac{\sigma_{i}^{2}}{\sigma_{n}^{2}}\right)\right| \frac{\tau_{i}^{2}}{\sigma_{i}^{2}}\left|S_{i}-E S_{i}\right| \\
& \leq \sum_{i=1}^{m_{n}(\varepsilon)}\left|f\left(\frac{\sigma_{i}^{2}}{\sigma_{n}^{2}}\right)\right| \frac{\tau_{i}^{2}}{\sigma_{n}^{2}} \frac{\sigma_{i}}{\sigma_{n}} \longrightarrow \int_{0}^{\varepsilon}|f(x)| \sqrt{x} d x, \\
& \text { as } n \longrightarrow \infty, \text { for fixed } \varepsilon>0 .
\end{aligned}
$$

Since from the definition of $m_{n}(t)$ we have $\sigma_{m_{n}(\varepsilon)}^{2} / \sigma_{n}^{2} \rightarrow$ $\varepsilon$, therefore, by the integrability of the function $f(x) \sqrt{x}$ and Lemma 4(a) we get

$$
\lim _{\varepsilon \rightarrow 0} \limsup _{n \rightarrow \infty} d_{\mathrm{KF} D[0,1]}\left(Y_{n}(t), Y_{n, \varepsilon}(t)\right)=0 .
$$
$C[0,1]$

Let us define a continuous mapping $H_{\varepsilon}: D[0,1] \rightarrow$

$$
H_{\varepsilon}(g)(t)= \begin{cases}0, & 0 \leq t<\varepsilon \\ \int_{\varepsilon}^{t} g(x) f(x) d x, & \varepsilon<t \leq 1 .\end{cases}
$$

The above integral is properly defined since $g$ is bounded and $f \in \mathscr{C}^{1}[\varepsilon, 1]$. In the next step we shall prove that

$$
H_{\varepsilon}\left(W_{n}\right)(t)=Y_{n, \mathcal{\varepsilon}}(t)+B_{n}(t)
$$

where $B_{n} \stackrel{P}{\rightarrow} 0$ in $D[0,1]$, as $n \rightarrow \infty$ and

$$
W_{n}(t)=\sum_{k=1}^{m_{n}(t)+1} \frac{\left(X_{k}-\mu_{k}\right)}{\sigma_{n}}
$$

At first let us observe that

$$
\begin{aligned}
& E \max _{0 \leq t \leq \varepsilon}\left|H_{\varepsilon}\left(W_{n}\right)(t)-Y_{n, \varepsilon}(t)\right| \\
& =\frac{1}{\sigma_{n}}\left|f\left(\frac{\sigma_{m_{n}(\varepsilon)+1}^{2}}{\sigma_{n}^{2}}\right)\right| \frac{\tau_{m_{n}(\varepsilon)+1}^{2}}{\sigma_{n}^{2}} E\left|S_{m_{n}(\varepsilon)+1}-E S_{m_{n}(\varepsilon)+1}\right| \\
& \quad \leq \frac{\sigma_{m_{n}(\varepsilon)+1}}{\sigma_{n}} \sup _{\varepsilon \leq x \leq 1}|f(x)| \frac{\max _{1 \leq i \leq n} \tau_{i}^{2}}{\sigma_{n}^{2}} \longrightarrow 0, \text { as } n \longrightarrow \infty
\end{aligned}
$$

by the Feller condition (18).

In the case $\varepsilon<t \leq 1$, let us calculate the integral

$$
\begin{aligned}
H_{\varepsilon}\left(W_{n}\right)(t)= & \int_{\varepsilon}^{t} f(x) W_{n}(x) d x \\
= & -\int_{\sigma_{m_{n}(\varepsilon)}^{2} / \sigma_{n}^{2}}^{\varepsilon} f(x) W_{n}(x) d x \\
& -\int_{t}^{\sigma_{m_{n}(t)+1}^{2} / \sigma_{n}^{2}} f(x) W_{n}(x) d x \\
& +\sum_{i=m_{n}(\varepsilon)+1}^{m_{n}(t)+1} \int_{\sigma_{i-1}^{2} / \sigma_{n}^{2}}^{\sigma_{i}^{2} / \sigma_{n}^{2}} f(x) W_{n}(x) d x \\
= & T_{n}^{\prime}(t)+T_{n}^{\prime \prime}(t)+T_{n}^{\prime \prime \prime}(t), \text { say. }
\end{aligned}
$$

In fact $T_{n}^{\prime}(t)$ does not depend on $t$, thus

$$
\begin{aligned}
E \max _{\varepsilon \leq t \leq 1}\left|T_{n}^{\prime}(t)\right| & \\
= & E\left|-\int_{\sigma_{m_{n}(\varepsilon)}^{2} / \sigma_{n}^{2}}^{\varepsilon} f(x) W_{n}(x) d x\right| \leq\left|\varepsilon-\frac{\sigma_{m_{n}(\varepsilon)}^{2}}{\sigma_{n}^{2}}\right| \\
& \times \sup _{\sigma_{m_{n}(\varepsilon)}^{2} / \sigma_{n}^{2} \leq x \leq 1}|f(x)| E \frac{\left|S_{m_{n}(\varepsilon)+1}-E S_{m_{n}(\varepsilon)+1}\right|}{\sigma_{n}} \longrightarrow 0,
\end{aligned}
$$

as $n \rightarrow \infty$, since $\sigma_{m_{n}(\varepsilon)}^{2} / \sigma_{n}^{2} \rightarrow \varepsilon$ and $E\left|S_{m_{n}(\varepsilon)+1}-E S_{m_{n}(\varepsilon)+1}\right| \leq$ $\sigma_{m_{n}(\varepsilon)+1} \leq \sigma_{n}$. 
Let us find the bounds for the second term $T_{n}^{\prime \prime}(t)$ :

$$
\begin{aligned}
\left|T_{n}^{\prime \prime}(t)\right| & =\left|\int_{t}^{\sigma_{m_{n}(t)+1}^{2} / \sigma_{n}^{2}} f(x) W_{n}(x) d x\right| \\
& \leq \frac{\max _{1 \leq i \leq n} \tau_{i}^{2}}{\sigma_{n}^{2}} \sup _{\varepsilon \leq x \leq 1}|f(x)| \frac{\left|S_{m_{n}(t)+1}-E S_{m_{n}(t)+1}\right|}{\sigma_{n}} .
\end{aligned}
$$

Further, for any $\delta>0$, we have

$$
\begin{aligned}
& P\left(\max _{\varepsilon \leq t \leq 1}\left|T_{n}^{\prime \prime}(t)\right|>\delta\right) \\
& \quad \leq P\left(\frac{\max \tau_{i}^{2}}{\sigma_{n}^{3}} \sup _{\varepsilon \leq x \leq 1}|f(x)| \max _{\varepsilon \leq t \leq 1}\left|S_{m_{n}(t)+1}-E S_{m_{n}(t)+1}\right|>\delta\right) \\
& \quad \leq P\left(\frac{\max \tau_{i}^{2}}{\sigma_{n}^{3}} \sup _{\varepsilon \leq x \leq 1}|f(x)| \max _{1 \leq i \leq n}\left|S_{i}-E S_{i}\right|>\delta\right) \\
& \quad \leq\left(\frac{\max \tau_{i}^{2}}{\sigma_{n}^{2}}\right)^{2}\left(\sup _{\varepsilon \leq x \leq 1}|f(x)|\right)^{2} \frac{1}{\delta^{2}} \longrightarrow 0, \quad \text { as } n \longrightarrow \infty,
\end{aligned}
$$

by the Kolmogorov inequality and the Feller condition (18).

Finally, let $n$ be sufficiently large, that is, such that $\sigma_{m_{n}(\varepsilon)}^{2} / \sigma_{n}^{2} \geq \varepsilon / 2$, then

$$
\begin{aligned}
\max _{\varepsilon \leq t \leq 1} & \left|T_{n}^{\prime \prime \prime}(t)-Y_{n, \varepsilon}(t)\right| \\
\leq & \max _{\varepsilon \leq t \leq 1} \frac{1}{\sigma_{n_{i=m_{n}}(\varepsilon)+1}} \sum_{m_{n}(t)+1}\left|S_{i}-E S_{i}\right| \\
& \times\left|\int_{\sigma_{i-1}^{2} / \sigma_{n}^{2}}^{\sigma_{i}^{2} / \sigma_{n}^{2}} f(x) d x-f\left(\frac{\sigma_{i}^{2}}{\sigma_{n}^{2}}\right) \frac{\tau_{i}^{2}}{\sigma_{n}^{2}}\right| \\
\leq & \frac{\max _{1 \leq i \leq n}\left|S_{i}-E S_{i}\right|}{\sigma_{n}} \\
& \times \max _{\varepsilon \leq t \leq 1} \sum_{i=m_{n}(\varepsilon)+1}^{m_{n}(t)+1}\left|\int_{\sigma_{i-1}^{2} / \sigma_{n}^{2}}^{\sigma_{i}^{2} / \sigma_{n}^{2}}\right| f(x)-f\left(\frac{\sigma_{i}^{2}}{\sigma_{n}^{2}}\right)|d x| \\
\leq & \frac{\max _{1 \leq i \leq n}\left|S_{i}-E S_{i}\right|}{\sigma_{n}} \sum_{i=1}^{n} c_{f, \varepsilon}\left(\frac{\tau_{i}^{2}}{\sigma_{n}^{2}}\right)^{2} \\
\leq & \frac{\max _{1 \leq i \leq n}\left|S_{i}-E S_{i}\right|}{\sigma_{n}} \frac{\max _{\tau_{i}^{2}}}{\sigma_{n}^{2}} c_{f, \varepsilon},
\end{aligned}
$$

where $c_{f, \varepsilon}=\max _{\varepsilon / 2 \leq x \leq 1}\left|f^{\prime}(x)\right|$. Thus, again by the Kolmogorov inequality and the Feller condition, we get

$$
\begin{aligned}
& P\left(\max _{\varepsilon \leq t \leq 1}\left|T_{n}^{\prime \prime \prime}(t)-Y_{n, \varepsilon}(t)\right|>\delta\right) \\
& \leq P\left(\frac{\max _{1 \leq i \leq n}\left|S_{i}-E S_{i}\right|}{\sigma_{n}} \frac{\max \tau_{i}^{2}}{\sigma_{n}^{2}} c_{f, \varepsilon}>\delta\right) \\
& \leq\left(\frac{\max \tau_{i}^{2}}{\sigma_{n}^{2} \delta} c_{f, \varepsilon}\right)^{2} \longrightarrow 0, \quad \text { as } n \longrightarrow \infty .
\end{aligned}
$$

Consequently, from (30), (32), (34), and (36) the statement (28) follows.

To end the proof, let us note that for the process $W_{n}(t)$ defined by (29), we have $W_{n}(t) \stackrel{d}{\rightarrow} W(t)$ in $D[0,1]$, by the result of Prohorov (see Problem 1, page 77 in [7]). In fact, Prohorov considered a broken-line process $X_{n}(t)$ and its convergence in $C[0,1]$, but

$$
\sup _{0 \leq t \leq 1}\left|W_{n}(t)-X_{n}(t)\right| \leq \frac{\max _{1 \leq i \leq n}\left|X_{i}-E X_{i}\right|}{\sigma_{n}} \stackrel{P}{\longrightarrow} 0,
$$

from the Lindeberg condition (9). Thus, by the continuity of $H_{\varepsilon}$, we get

$$
H_{\varepsilon}\left(W_{n}\right)(t) \stackrel{d}{\longrightarrow} H_{\varepsilon}(W)(t), \quad \text { in } D[0,1], \text { as } n \longrightarrow \infty .
$$

Furthermore,

$$
\begin{array}{r}
\sup _{0 \leq t \leq 1}\left|H_{\varepsilon}(W)(t)-\int_{0}^{t} f(x) W(x) d x\right| \\
=\sup _{0 \leq t \leq \varepsilon}\left|\int_{0}^{t} f(x) W(x) d x\right| \\
\leq \sup _{0<x \leq \varepsilon} \frac{|W(x)|}{\sqrt{x \log (1 / x)}} \cdot \int_{0}^{\varepsilon}|f(x)| \sqrt{x \log \left(\frac{1}{x}\right)} d x \longrightarrow 0, \\
\text { almost surely as } \varepsilon \longrightarrow 0^{+},
\end{array}
$$

by (5) and the properties of the modulus of continuity of the Wiener process, that is,

$$
\limsup _{\varepsilon \rightarrow 0^{+}} \sup _{0<x \leq \varepsilon} \frac{|W(x)|}{\sqrt{x \log (1 / x)}} \leq \sqrt{2} \text { almost surely; }
$$

see formula (7) on page 534 in [6]. 
For short, let us put $H(W)(t)=\int_{0}^{t} f(x) W(x) d x$. Then, with the notation introduced in Lemma 4 , we get

$$
\begin{aligned}
& d_{\mathrm{LP} D[0,1]}\left(Y_{n} ; H(W)\right) \\
& \leq d_{\mathrm{LP} D[0,1]}\left(Y_{n} ; Y_{n, \varepsilon}\right)+d_{\mathrm{LP} D[0,1]}\left(Y_{n, \varepsilon} ; H_{\varepsilon}\left(W_{n}\right)\right) \\
&+d_{\mathrm{LP} D[0,1]}\left(H_{\varepsilon}\left(W_{n}\right) ; H_{\varepsilon}(W)\right) \\
&+d_{\mathrm{LP} D[0,1]}\left(H_{\varepsilon}(W) ; H(W)\right) \\
& \leq d_{\mathrm{KF} D[0,1]}\left(Y_{n} ; Y_{n, \varepsilon}\right)+d_{\mathrm{KF}[0,1]}\left(Y_{n, \varepsilon} ; H_{\varepsilon}\left(W_{n}\right)\right) \\
&+d_{\mathrm{LP} D[0,1]}\left(H_{\varepsilon}\left(W_{n}\right) ; H_{\varepsilon}(W)\right) \\
&+d_{\mathrm{KF} D[0,1]}\left(H_{\varepsilon}(W) ; H(W)\right) .
\end{aligned}
$$

Now, (23) follows from (26), (28), (38), and (39) and the proof is completed.

\section{References}

[1] B. C. Arnold and J. A. Villaseñor, "The asymptotic distributions of sums of records," Extremes, vol. 1, no. 3, pp. 351-363, 1999.

[2] G. Rempała and J. Wesołowski, "Asymptotics for products of sums and $U$-statistics," Electronic Communications in Probability, vol. 7, pp. 47-54, 2002.

[3] L.-X. Zhang and W. Huang, "A note on the invariance principle of the product of sums of random variables," Electronic Communications in Probability, vol. 12, pp. 51-56, 2007.

[4] K. M. Kosiński, "On the functional limits for sums of a function of partial sums," Statistics \& Probability Letters, vol. 79, no. 13, pp. 1522-1527, 2009.

[5] P. Matuła and I. Stępień, "Weak convergence of products of sums of independent and non-identically distributed random variables," Journal of Mathematical Analysis and Applications, vol. 353, no. 1, pp. 49-54, 2009.

[6] G. R. Shorack and J. A. Wellner, Empirical Processes with Applications to Statistics, John Wiley \& Sons Inc., New York, NY, USA, 1986.

[7] P. Billingsley, Convergence of Probability Measures, John Wiley \& Sons, New York, NY, USA, 1968.

[8] R. M. Dudley, Real Analysis and Probability, Cambridge Studies in Advanced Mathematics, Cambridge University, Cambridge, Uk, 2002.

[9] W. J. Kaczor and M. T. Nowak, Problems in Mathematical Analysis. I, American Mathematical Society, Providence, RI, USA, 2000. 


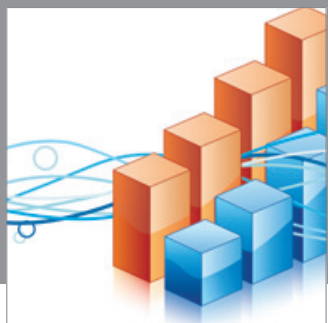

Advances in

Operations Research

mansans

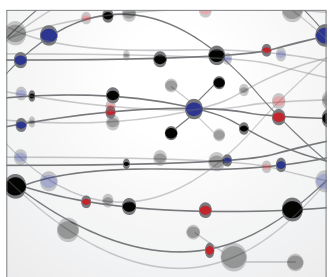

The Scientific World Journal
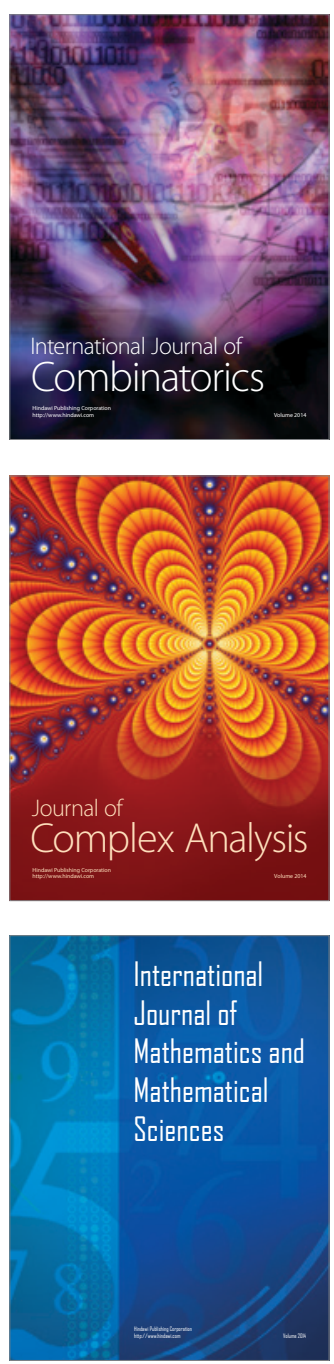
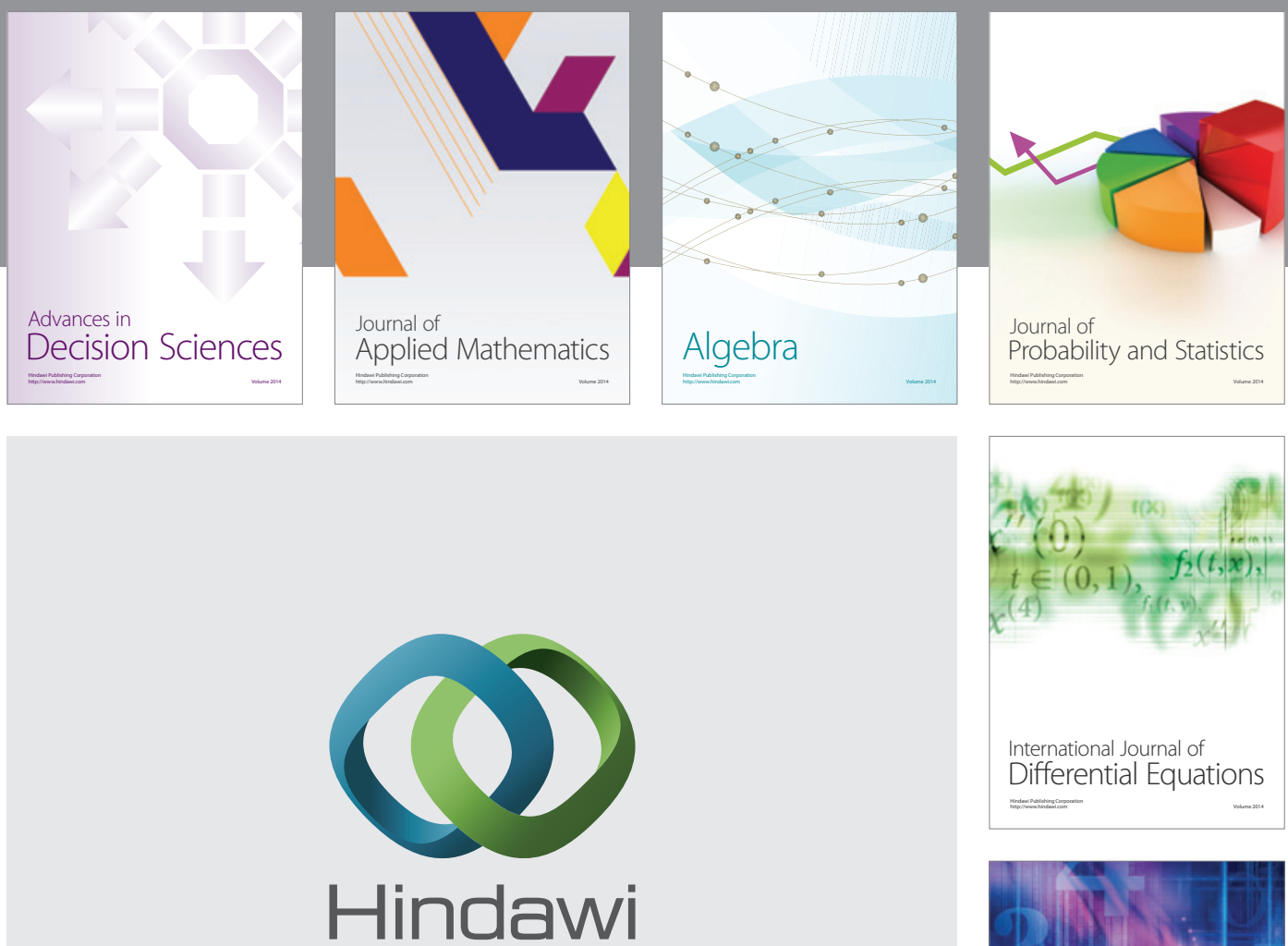

Submit your manuscripts at http://www.hindawi.com
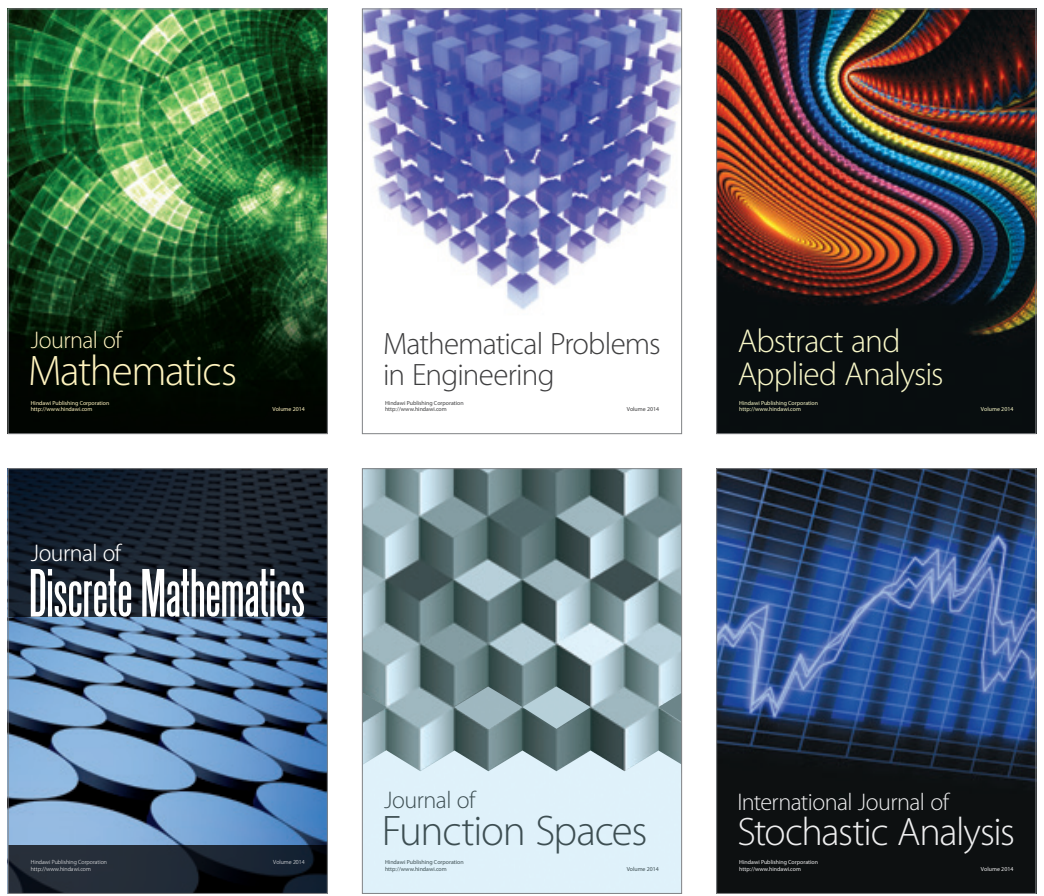

Journal of

Function Spaces

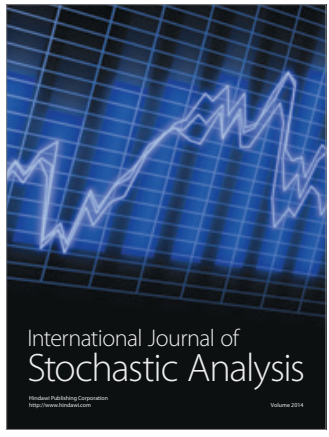

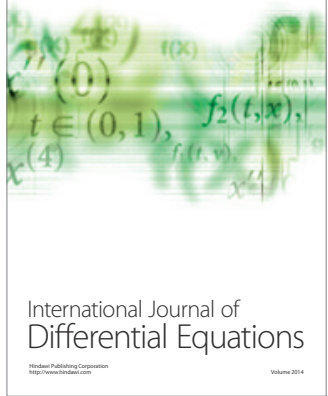
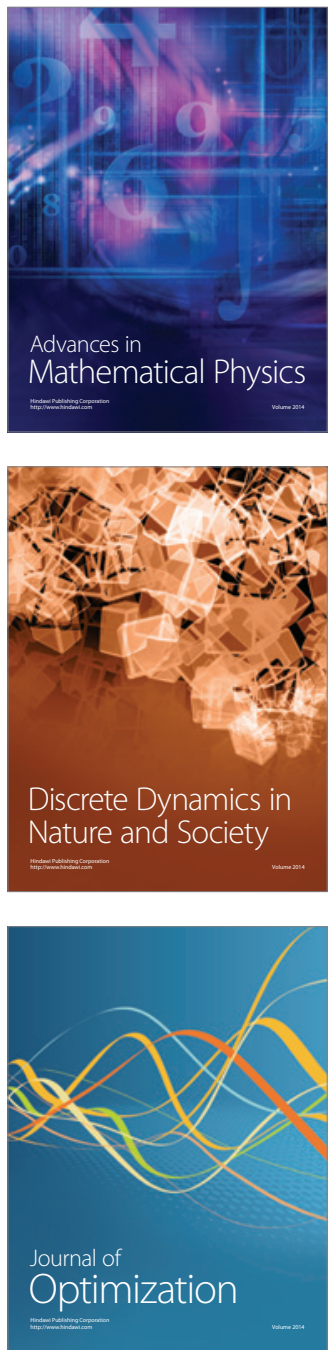